\title{
Efficient Analysis of Phased Arrays of Microstrip Patches Using a Hybrid Generalized Forward Backward Method/Green's Function Technique With a DFT Based Acceleration Algorithm
}

\author{
Onur Bakır, Student Member, IEEE, Özlem Aydın Çivi, Senior Member, IEEE, Vakur B. Ertürk, Member, IEEE, \\ and Hsi-Tseng Chou, Senior Member, IEEE
}

\begin{abstract}
A hybrid method based on the combination of generalized forward backward method (GFBM) and Green's function for the grounded dielectric slab together with the acceleration of the combination via a discrete Fourier transform (DFT) based algorithm is developed for the efficient and accurate analysis of electromagnetic radiation/scattering from electrically large, irregularly contoured two-dimensional arrays consisting of finite number of probe-fed microstrip patches. In this method, unknown current coefficients corresponding to a single patch are first solved by a conventional Galerkin type hybrid method of moments (MoM)/Green's function technique that uses the grounded dielectric slab's Green's function. Because the current distribution on the microstrip patch can be expanded using an arbitrary number of subsectional basis functions, the patch can have any shape. The solution for the array currents is then found through GFBM, where it sweeps the current computation element by element. The computational complexity of this method, which is originally $O\left(N_{\text {tot }}^{2}\right)\left(N_{\text {tot }}\right.$ being the total number of unknowns) for each iteration, is reduced to $O\left(N_{\text {tot }}\right)$ using a DFT based acceleration algorithm making use of the fact that array elements are identical and the array is periodic. Numerical results in the form of array current distribution are given for various sized arrays of probe-fed microstrip patches with elliptical and/or circular boundaries, and are compared with the conventional MoM results to illustrate the efficiency and accuracy of the method.
\end{abstract}

Index Terms-Discrete Fourier transform (DFT), finite arrays, generalized forward backward method (GFBM), method of moments (MoM).

\section{INTRODUCTION}

$\mathbf{M}$ AJORITY of commercial and military applications require phased arrays fabricated using the integrated circuit technology. However, a great portion of available design

Manuscript received May 4, 2007; revised October 2, 2007. This work was supported in part by the Turkish Scientific and Technological Research Council (TÜBITAK) under Grant EEEAG-104E044 and the Turkish Academy of Sciences (TÜBA)-GEBİP.

O. Bakır was with the Department of Electrical and Electronics Engineering, Bilkent University, TR-06800, Bilkent, Ankara, Turkey. He is now with the Department of Electrical and Computer Engineering, University of Michigan, Ann Arbor, MI 48109 USA (e-mail: onurb@umich.edu).

Ö. A. Çivi is with the Department of Electrical and Electronics Engineering Middle East Technical University, Inonu Bulvari, 06531 Ankara, Turkey.

V. B. Ertürk is with the Department of Electrical and Electronics Engineering, Bilkent University, TR-06800, Bilkent, Ankara, Turkey.

H.-T. Chou is with the Department of Electrical Engineering, Yuan Ze University, Chung Li 320, Taiwan.

Color versions of one or more of the figures in this paper are available online at http://ieeexplore.iee.org.

Digital Object Identifier 10.1109/TAP.2008.923314 and analysis software, in particular computer-aided design (CAD) tools, may suffer from the memory storage requirements and computational cost when the number of elements in the array increases rapidly. Therefore, as an alternative to the numerical approaches such as finite elements or finite difference time domain techniques [1]-[4], various integral equation (IE) based method of moments (MoM) solutions, (which use the grounded dielectric slab's Green's function as the kernel of the integral equation) have been developed to be implemented in CAD packages to perform the full wave analysis of planar, large, finite printed phased arrays accurately and efficiently [5]-[21].

Although the infinite array based approach [5]-[7], which convolves the Floquet-based infinite array solution with the array aperture distribution, has attracted attention due to its simplicity and efficiency, it can not fully capture the finite array truncation effects, which affects the accuracy of both the element input impedance and the array radiation/scattering patterns. Therefore, to improve the efficiency of the rigorous "element-by-element" MoM approach ([8], [9]) has become the center of attention. In [10]-[12], the discrete Fourier transform (DFT) technique has been used in conjunction with the MoM in such a way that the DFT coefficients of the array currents are formulated to be the new unknowns of the MoM matrix-equation rather than the array currents yielding a great reduction in the number of unknowns. On the other hand, in [13] a DFT based preconditioner is constructed and used in an iterative MoM to significantly improve the convergence rate of the iterative solution. Similarly, a DFT based acceleration algorithm is developed in [14] and used in conjunction with stationary and non-stationary iterative MoM solutions in [15]-[17] to obtain a great reduction in both the computational complexity and storage requirements. Besides the DFT technique, fast Fourier transform (FFT) algorithm is also combined with conjugate gradient based iterative techniques in [18], [19] to obtain fast full-wave solvers for finite arrays. Recently, a synthesis of the sparse matrix/adaptive integral method (SM/AIM) and the multiresolution (MR) approach is presented for the analysis of electrically large finite arrays [20], where the MR technique serves as an efficient preconditioner for the SM/AIM. In addition to these aforementioned studies, hybrid combinations of uniform theory of diffraction (UTD) with MoM have been presented for the fast analysis of electrically large, planar periodic finite free-standing [22], [23] and printed [21] dipole 
arrays. In these approaches, regardless of the array size a fixed number of unknowns (very few compared to the array size) is sufficient in the MoM analysis to accurately predict array currents. However, such approaches have not been extended yet to arrays having elements other than dipoles.

In this paper, a hybrid combination of the generalized forward backward method (GFBM) [24] with the grounded dielectric slab's Green's function accelerated with a DFT based algorithm, namely GFBM/Green's function-DFT method, is introduced for the fast and accurate analysis of finite phased arrays of probe-fed microstrip patches on planar grounded dielectric slabs. The method presented here is actually the generalized form of the method presented in [14]-[17], and can handle any finite, irregularly contoured, one or two dimensional, periodic, free-standing and/or printed arrays as long as the array elements are identical. The proposed method starts by finding the current coefficients of a single patch by employing a conventional Galerkin type hybrid MoM/Green's function method. The solution for the array currents is then found through GFBM, where it sweeps the current computation element by element. Because the current distribution on each element can be expanded using an arbitrary number of subsectional basis functions, elements of the array can, in fact, have any shape. The computational complexity of this method, which is originally $O\left(N_{\text {tot }}^{2}\right)\left(N_{\text {tot }}\right.$ being the total number of unknowns) for each iteration, is reduced to $O\left(N_{\text {tot }}\right)$. This is achieved by combining a slightly modified version of the DFT based acceleration algorithm developed in [14] with the fact that array elements are identical and the array is periodic. Briefly, the DFT based acceleration algorithm divides the contributing elements into "strong" and "weak" interaction groups for a receiving element in the GFBM. Contributions from the strong group are obtained by the conventional element-by-element computation to assure the fundamental accuracy, whereas contributions coming from the weak region are obtained based on the DFT representation of the array current. In general, only a few significant DFT terms are sufficient to provide accurate results as they provide minor corrections to the solution in contrast to the dominating strong group. On the other hand, to handle more basis functions per elements, subarrays are formed among the $r$ th basis functions of elements ( $r=1,2, \ldots, N_{b}, N_{b}$ being the total number of basis functions per element) using the periodicity of the array and array elements being identical. Then, contributions coming from the weak region of the actual array is calculated by superposing the weak region contributions of each subarray using the corresponding DFT coefficient.

In Section II, the geometry and a brief information regarding the conventional hybrid MoM/Green's function solution for the finite phased arrays of probe-fed microstrip patches on planar grounded dielectric slabs are presented. Hence, this section may provide insight to the proposed GFBM/Green's function-DFT solution as well as it serves as a reference solution used to assess the efficiency and accuracy of GFBM/Green's function-DFT method. Section III provides the detailed description of the GFBM/Green's function-DFT formulation. Numerical results in the form of array current distribution are provided for various arrays using the proposed GFBM/Green's function-DFT method, and compared with the conventional MoM/Green's function results in Section IV. An $e^{j \omega t}$ time dependence is assumed and suppressed throughout this paper.

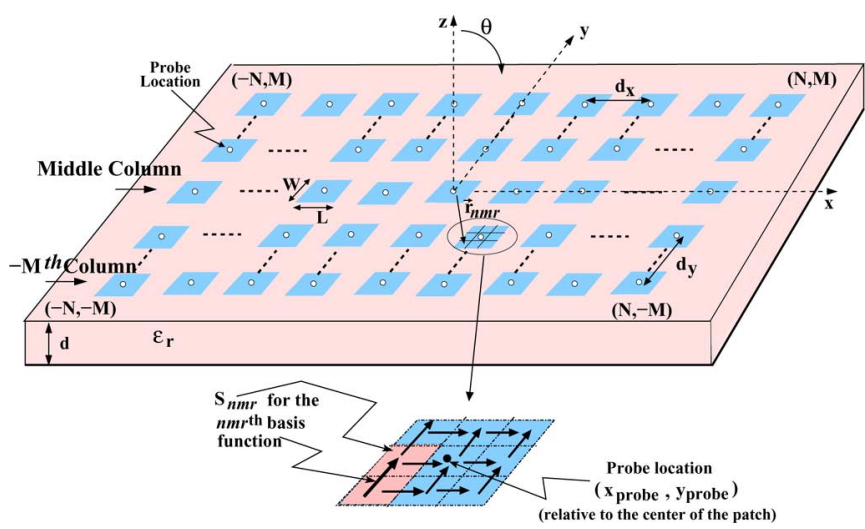

Fig. 1. Geometry of a finite, periodic, planar array of $(2 N+1) \times(2 M+1)$ probe-fed microstrip patches on a grounded dielectric slab; and MoM model of a single patch. Each arrow represents a piecewise sinusoidal basis function.

\section{FORMULATION}

\section{A. Geometry}

The geometry of a finite, periodic, planar array of $N_{\text {element }}\left[N_{\text {element }}=(2 N+1) \times(2 M+1)\right]$ identical printed microstrip patches is depicted in Fig. 1. The microstrip patches are printed on the dielectric-air interface $(z=0)$ of a grounded dielectric slab, which has a thickness $d$ and a relative dielectric constant $\epsilon_{r}>1$. Each patch is assumed to be probe-fed with an ideal current source, has a length $L$, width $W$, and is uniformly spaced from its neighbors by distances $d_{x}$ and $d_{y}$ in the $\hat{x}$ and $\hat{y}$ directions, respectively.

The configuration of Fig. 1 is chosen because it provides simplicity for illustrating the development of the present GFBM/ Green's function-DFT method. However, this approach can be extended to deal with more complex array elements and/or with arbitrarily contoured arrays such as elliptical or circular ones.

\section{B. Conventional Hybrid MoM/Green's Function Method Solution for Phased Arrays}

An electric field integral equation (EFIE) is formed by enforcing the boundary condition that the total tangential electric field should vanish on the microstrip patch surfaces. Then, the conventional hybrid MoM/Green's function solution starts with an expansion of the unknown induced array surface current, $\mathbf{J}^{s}(\mathbf{r})$, in terms of a finite set of subsectional basis functions

$$
\mathbf{J}^{s}(\mathbf{r})=\sum_{n=-N}^{N} \sum_{m=-M}^{M} \sum_{r=1}^{N_{b}} A_{n m r} \mathbf{J}_{n m r}(\mathbf{r})
$$

where

$$
\mathbf{J}_{n m r}(\mathbf{r})= \begin{cases}f_{n m i}(\mathbf{r}) \hat{x}, & i=1, \ldots, I \text { on the } n m \text { th patch } \\ f_{n m j}(\mathbf{r}) \hat{y}, & j=1, \ldots, J \text { on the } n m \text { th patch }\end{cases}
$$

with $\mathbf{r}$ as the position vector of $\mathbf{J}_{n m r}, N_{b}=I+J$ being the total number of subsectional basis functions per microstrip patch and $f_{n m i}(\mathbf{r})$ [and $f_{n m j}(\mathbf{r})$ ] being piecewise sinusoidal along the direction of the current and constant in the direction perpendicular 
to the current. Finally, $A_{n m r}$ are the unknowns to be computed and

$$
\mathbf{J}_{n m r}(\mathbf{r}) \neq 0 \text { only if } \mathbf{r} \in S_{n m r}
$$

with $S_{n m r}$ being the area occupied by the $n m r$ th piecewise sinusoidal basis function as illustrated in Fig. 1 .

Selecting the testing functions, $w_{p q s}(-N \leq p \leq N,-M \leq$ $q \leq M, 1 \leq s \leq N_{b}$ ), the same as the basis functions (Galerkin procedure), the following matrix-equation is obtained:

$$
\overline{\mathbf{Z}} \cdot \mathbf{I}=\mathbf{V} \text {. }
$$

In (4), I is the $N_{\text {tot }} \times 1\left(N_{\text {tot }}=N_{\text {element }} N_{b}\right)$ column vector consisting of the unknown current amplitude $A_{n m r}$ of the $r$ th basis function belonging to the $n m$ th patch and $\overline{\mathbf{Z}}$ is the $N_{\text {tot }} \times N_{\text {tot }}$ impedance matrix of the array with elements $Z_{n m r, p q s}$, which denotes the mutual impedance between the $r$ th basis function of the $n m$ th patch and $s$ th testing function of the $p q$ th patch. It is explicitly given by

$$
\begin{aligned}
Z_{n m r, p q s}= & \int_{S_{p q s}} d S_{p q s} \int_{S_{n m r}} d S_{n m r}^{\prime} f_{p q s}(\mathbf{r}) \hat{v} \\
& \cdot \overline{\mathbf{G}}\left(\mathbf{r} \mid \mathbf{r}^{\prime}\right) \cdot \hat{u} f_{n m r}\left(\mathbf{r}^{\prime}\right) ; \hat{u}, \hat{v}=\hat{x} \text { or } \hat{y}
\end{aligned}
$$

(with $\mathbf{r}$ and $\mathbf{r}^{\prime}$ being the position vectors on the $p q s$ th and $n m r$ th current modes, respectively). On the other hand, $\mathbf{V}$ at the right hand side of (4) is the $N_{\text {tot }} \times 1$ column vector related to the excitation of the array. Assuming an ideal probe (a unit current source at the probe location) located at the $\left(x_{\text {probe }}, y_{\text {probe }}\right)$ position of any of the patches, a typical element of $\mathbf{V}$ is given by

$$
\begin{aligned}
V_{p q s}=e^{-j \beta_{x} p d_{x}} & e^{-j \beta_{y} q d_{y}} \int_{S_{p q s}} d S_{p q s} \\
& \times\left[\int_{-d}^{0} d z G_{z v}\left(x_{\text {probe }}, y_{\text {probe }}, z \mid \mathbf{r}\right)\right] f_{p q s}(\mathbf{r})
\end{aligned}
$$

with

$$
\beta_{x}=k_{0} \sin \theta \cos \phi ; \quad \beta_{y}=k_{0} \sin \theta \sin \phi
$$

where $(\theta, \phi)$ is the scan direction of the main beam and $k_{0}$ is the free-space propagation constant. Finally, $G_{u v}\left(\mathbf{r} \mid \mathbf{r}^{\prime}\right)$ in (5) and $G_{z v}\left(x_{\text {probe }}, y_{\text {probe }}, z \mid \mathbf{r}\right)$ in (6) are the corresponding components of the spatial domain representation of the dyadic Green's function, $\mathbf{G}\left(\mathbf{r} \mid \mathbf{r}^{\prime}\right)$, for the infinite grounded dielectric slab [25]-[28].

\section{Review of Generalized Forward Backward Method (GFBM) For Phased Arrays}

Rather than using direct solution methods such as Gaussian elimination or LU-decomposition to solve (4), one can employ GFBM [24] as an iterative algorithm, which yields nearly the same accuracy and converges rapidly. To employ GFBM to (4),

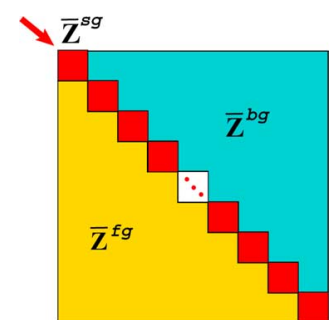

Fig. 2. Decomposition of $\overline{\mathbf{Z}}$ in the GFBM algorithm; $\overline{\mathbf{Z}}^{s g}$ : strong group, $\overline{\mathbf{Z}}^{\text {fg }}$ : forward group, $\overline{\mathbf{Z}}^{b g}$ : backward group.

first the current vector, $\mathbf{I}$, is decomposed into its forward, $\mathbf{I}^{f}$, and backward, $\mathbf{I}^{b}$, components as

$$
\mathbf{I}=\mathbf{I}^{f}+\mathbf{I}^{b}
$$

and the impedance matrix, $\overline{\mathbf{Z}}$, is expressed as

$$
\overline{\mathbf{Z}}=\overline{\mathbf{Z}}^{s g}+\overline{\mathbf{Z}}^{f g}+\overline{\mathbf{Z}}^{b g}
$$

where $\overline{\mathbf{Z}}^{s g}$ is formed by the block diagonal matrices of $\overline{\mathbf{Z}}$ corresponding to the impedance matrix of a single patch, whereas $\overline{\mathbf{Z}}^{f g}$ and $\overline{\mathbf{Z}}^{b g}$ are the lower and upper triangular parts of $\overline{\mathbf{Z}}$ with $\overline{\mathbf{Z}}^{s g}$ subtracted, respectively, as illustrated in Fig. 2 . Then, the original matrix-equation, given by (4), is transformed to

$$
\begin{aligned}
& \overline{\mathbf{Z}}^{s g} \mathbf{I}^{f}=\mathbf{V}-\overline{\mathbf{Z}}^{f g}\left(\mathbf{I}^{f}+\mathbf{I}^{b}\right) \\
& \overline{\mathbf{Z}}^{s g} \mathbf{I}^{b}=-\overline{\mathbf{Z}}^{b g}\left(\mathbf{I}^{f}+\mathbf{I}^{b}\right) .
\end{aligned}
$$

Initializing $\mathbf{I}^{b}$ to zero at the first iteration, (10) is solved for $\mathbf{I}^{f}$ and the resultant $\mathbf{I}^{f}$ is used in (11) to solve for $\mathbf{I}^{b}$. Iterations are continued until convergence is provided; this requires, in general, three or four iterations. Since $N_{b}$ is small compared to $N_{\text {tot }}$, GFBM requires $O\left(N_{\text {tot }}^{2}\right)$ computational complexity and memory storage due to the repeated computations of $\overline{\mathbf{Z}}^{f g} \mathbf{I}$ and $\overline{\mathbf{Z}}^{b g} \mathbf{I}$.

\section{GFBM/Green's Function Method Accelerated With a DFT-Based Algorithm (GFBM/Green's Function-DFT)}

The GFBM/Green's function-DFT method for finite arrays of microstrip patches is, in fact, the generalized form of the method presented in [14]-[17] and is based on the compactness of the DFT spectrum of practical array currents.

Consider $\overline{\mathbf{Z}}^{f g} \mathbf{I}$ in (10), which represents contributions to a receiving antenna (i.e., receiving element) from the antennas in the front of this receiving antenna (forward sweep). Similar to [14] if we call these antennas as contributing elements, the DFT based acceleration algorithm starts by dividing these elements into strong and weak interaction groups as illustrated in Fig. 3 for a generic finite array of probe-fed microstrip patches with three basis functions per a single patch (each basis function is represented with a different color). These contributions can be 


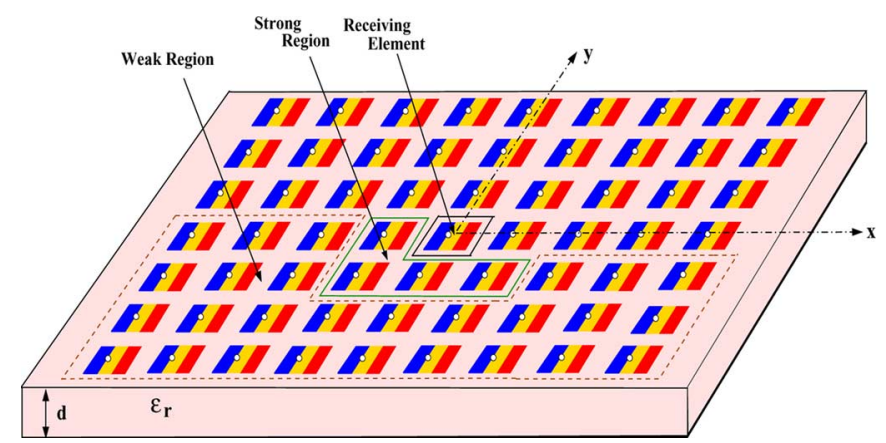

Fig. 3. Decomposition of a forward-sweep contribution region of the receiving element into strong and weak interaction groups.

expressed as

$$
\begin{aligned}
{[\overline{\mathbf{Z}}]_{p q s}^{f g}=\sum_{n, m, r \in \text { strong }} A_{n m r} Z_{n m r, p q s} } & \\
& +\sum_{n, m, r \in \text { weak }} A_{n m r} Z_{n m r, p q s}
\end{aligned}
$$

where strong and weak groups are denoted by strong and weak, respectively.

Elements that are distanced within a few wavelengths (usually $1 \lambda_{0}$ or $1.5 \lambda_{0}, \lambda_{0}$ : free-space wavelength, is enough) from receiving elements form the strong group and the total number of elements in this group is denoted by $N_{s}$. Its size is fixed for most of the receiving elements during the forward sweep and is very small compared to the total number of elements in the entire array. Because the contributions coming from the strong group have a major effect on the accuracy of the overall solution, they are obtained via an element-by-element computation, where a conjunction of the conventional hybrid MoM/Green's function method with the GFBM is used. This step mainly assures the fundamental accuracy for the final solution.

On the other hand, although contributions coming from the weak group provide only minor corrections to the overall solution, their evaluation without an acceleration algorithm would constitute the most time-consuming aspect of the GFBM solution procedure. Therefore, contributions coming from the weak region are obtained making use of the DFT representation of the induced entire array current distribution. However, because the current distribution on each element is expanded using an arbitrary number of subsectional basis functions, the acceleration algorithm presented in [14] is modified to handle more basis functions per elements provided that array elements are identical (i.e., each element has the same number of basis functions) and the array is periodic. Hence, if we consider only the first basis functions of all elements, a periodic subarray, just like the arrays described in [14]-[17], can be formed. The same is true for all other basis functions $\left(r=1,2, \ldots, N_{b}\right)$ as well. Then, using the DFT representation of $A_{n m r}$ (corresponding to the $r$ th subarray)

$$
\begin{aligned}
& A_{n m r}=e^{-j \beta_{x} n d_{x}} e^{-j \beta_{y} m d_{y}} \sum_{k=-N}^{N} \\
& \times \sum_{l=-M}^{M} B_{k l r} e^{-j 2 \pi k n / 2 N+1} e^{-j 2 \pi l m / 2 M+1}
\end{aligned}
$$

with $B_{k l r}$ being the coefficient of the $k l$ th DFT term ( $r$ th basis functions, i.e., $r$ th subarray) given by

$$
\begin{aligned}
B_{k l r}= & \frac{1}{(2 N+1)(2 M+1)} \sum_{n=-N}^{N} \sum_{m=-M}^{M} A_{n m r} \\
& \times e^{-j \beta_{x} n d_{x}} e^{-j \beta_{y} m d_{y}} e^{j 2 \pi k n / 2 N+1} e^{j 2 \pi l m / 2 M+1}
\end{aligned}
$$

the weak region contributions to the $s$ th basis function of the $p q$ th element is expressed as

$$
\begin{aligned}
{[\overline{\mathbf{Z}} \mathbf{I}]_{p q s ; \text { weak }}^{f g}=} & \sum_{r=1}^{N_{b}} \sum_{k=-N}^{N} \sum_{l=-M}^{M} B_{k l r} \\
& \times \sum_{n, m \in \text { weak }} Z_{n m r, p q s} e^{-j \beta_{x} n d_{x}} e^{-j \beta_{y} m d_{y}} \\
& \times e^{-j 2 \pi k n / 2 N+1} e^{-j 2 \pi l m / 2 M+1}
\end{aligned}
$$

Note that the DFT representation of practical large array current distribution is very compact. Only a few $B_{k l r}$ values are large compared to the rest. Hence, selecting only the significant DFT terms based on the criterion presented in [11], (15) can be rewritten as

$$
[\overline{\mathbf{Z}} \mathbf{I}]_{p q s ; \text { weak }}^{f g}=\sum_{r=1}^{N_{b}}\left[\sum_{k l \in Q} B_{k l r} C_{k l r, p q s}\right]
$$

where

$$
\begin{aligned}
C_{k l r, p q s}= & \sum_{n, m \in \text { weak }} Z_{n m r, p q s} e^{-j \beta_{x} n d_{x}} e^{-j \beta_{y} m d_{y}} \\
& \times e^{-j 2 \pi k n / 2 N+1} e^{-j 2 \pi l m / 2 M+1}
\end{aligned}
$$

and $Q$ is the set of significant DFT terms. The most important DFT term is $B_{00 r}$ (i.e., $k=0$ and $l=0$ for each subarray) which corresponds to the infinite array with uniform excitation. Selecting only this DFT term for finite arrays usually yields enough accuracy. However, for further accuracy one may choose a few more DFT terms for each subarray from the two orthogonal row $(k=0)$ and column $(l=0)$ (representing diffraction from the edges). More explanations on the selection of $Q$ can be found in [11] and [14]. It should be mentioned that because contributions coming from the weak group provide minor corrections to the overall solution, retaining only a few of the most significant DFT terms is sufficient from the accuracy point of view. As a result, $Q$ is actually a very small number (based on our extensive simulations, we noticed that taking more than two DFT terms (in addition to $B_{00 r}$ ) for each subarray does not improve the accuracy enough to justify the computational burden it produces).

$C_{k l r, p q s}$ in (17) denotes the contribution of the $k l$ th DFT term of the $r$ th basis function to the $s$ th basis function of the $p q$ th receiving element, and its efficient evaluation is very crucial to retain the computational complexity at $O\left(N_{\text {tot }}\right)$. Therefore, considering each periodic subarray one at a time (i.e., $r=s=$ $1,2, \ldots, N_{b}$ and each basis function becomes an element), the 


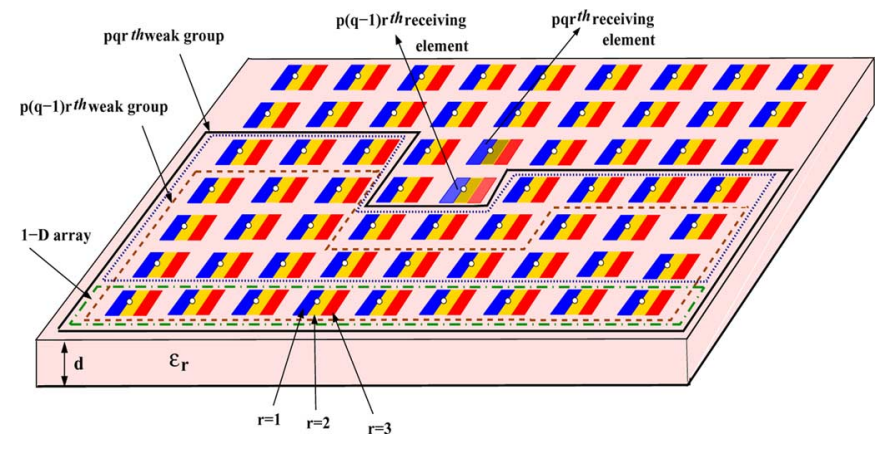

Fig. 4. The forward weak group corresponding to the $p q r$ th receiving element (the solid loop) is decomposed into two groups indicated by dotted and dash-dotted loops. The dotted loop is identical to the dashed loop, which is the forward weak group of $p(q-1) r$ th receiving element, except for a location shift, corresponding to a phase shift.

iterative calculation (apart from the usual GFBM iterations) presented in [14]-[16] is used. Considering only the forward sweep and assuming that the iterative procedure sweeps elements in the order of $p q=11,21,31, \ldots, 12,22, \ldots, 13,23, \ldots, C_{k l r, p q r}$ in (17) (denoted as $C_{k l r, p q r}^{f}$ ) is written as

$$
\begin{aligned}
C_{k l r, p q r}^{f}= & \sum_{m n \in W} Z_{n m r, p q r} e^{-j \beta_{x} n d_{x}} e^{-j \beta_{y} m d_{y}} \\
& \times e^{-j 2 \pi k n / 2 N+1} e^{-j 2 \pi l m / 2 M+1} \\
& +\sum_{n=-N}^{N} Z_{n(-M) r, p q r} e^{-j \beta_{x} n d_{x}} e^{-j \beta_{y}(-M) d_{y}} \\
& \times e^{-j 2 \pi k n / 2 N+1} e^{-j 2 \pi l(-M) / 2 M+1}
\end{aligned}
$$

where $W$ corresponds to the weak group when the $-M$ th column of the array is subtracted. Because the $Z_{n m r, p q r}$ in the first term of (18) depends only on $(p-n)$ and $(q-m)$, this term can be related to $C_{k l r, p(q-1) r}^{f}$ by

$$
\begin{aligned}
C_{k l r, p q r}^{f}= & C_{k l r, p(q-1) r}^{f} e^{-j \beta_{y} d_{y}} e^{-j 2 \pi l / 2 M+1} \\
& +\sum_{n=-N}^{N} Z_{n(-M) r, p q r} e^{-j \beta_{x} n d_{x}} e^{-j \beta_{y}(-M) d_{y}} \\
& \times e^{-j 2 \pi k n / 2 N+1} e^{-j 2 \pi l(-M) / 2 M+1}
\end{aligned}
$$

This relation is illustrated in Fig. 4 where the weak group of the pqrth receiving element (the solid loop in Fig. 4) is decomposed into two parts, the upper sub-group denoted by the dotted loop and the lower sub-group denoted by the dash-dotted loop which consists of the elements of the $-M$ th column. The upper sub-group of the pqrth element is identical to the weak group of the $p(q-1) r$ th element, shown with the dashed loop, except a location shift which corresponds to a phase shift. Since we have $N_{b}$ basis functions in each antenna element, this procedure is repeated $N_{b}$ times for $N_{b}$ subarrays as shown in Fig. 4 with $N_{b}=3$. It should also be noted that in the forward sweep $C_{k l r, p(q-1) r}^{f}$ is calculated before $C_{k l r, p q r}$ is interested.

Similarly, denoting the second term in (19) as $D_{k l r, p q r}^{f}$, which represents the contributions coming from a linearly phased one- dimensional array with the receiving element located far away from this array, it is iteratively calculated as

$$
\begin{aligned}
D_{k l r, p q r}^{f}= & \sum_{n=-N+1}^{N} Z_{n(-M) r, p q r} e^{-j \beta_{x} n d_{x}} e^{-j \beta_{y}(-M) d_{y}} \\
& \times e^{-j 2 \pi k n / 2 N+1} e^{-j 2 \pi l(-M) / 2 M+1} \\
& +Z_{(-N)(-M) r, p q r} e^{-j \beta_{x}(-N) d_{x}} e^{-j \beta_{y}(-M) d_{y}} \\
& \times e^{-j 2 \pi k(-N) / 2 N+1} e^{-j 2 \pi l(-M) / 2 M+1}
\end{aligned}
$$

and the first term can be related to $D_{k l r,(p-1) q r}^{f}$ by

$$
\begin{aligned}
D_{k l r, p q r}^{f}= & D_{k l r,(p-1) q r}^{f} e^{-j \beta_{x} d_{x}} e^{-j 2 \pi k / 2 N+1} \\
& -Z_{N(-M) r,(p-1) q r} e^{-j \beta_{x}(N+1) d_{x}} e^{-j \beta_{y}(-M) d_{y}} \\
& \times e^{-j 2 \pi k(N+1) / 2 N+1} e^{-j 2 \pi l(-M) / 2 M+1} \\
& +Z_{(-N)(-M) r, p q r} e^{-j \beta_{x}(-N) d_{x}} e^{-j \beta_{y}(-M) d_{y}} \\
& \times e^{-j 2 \pi k(-N) / 2 N+1} e^{-j 2 \pi l(-M) / 2 M+1}
\end{aligned}
$$

$C_{k l r, p q r}^{b}$, due to the 'backward' group can be found in a similar fashion by a backward sweep where the formulation will be in the same form of (18)-(21). Note that when $q$ is in the first few columns (i.e., $q \in-M$ or $q \in-M+1$ ), all contributing elements will be in its strong region. Hence, an element-by-element computation must be employed.

Besides, for the irregularly contoured two-dimensional arrays, the modification presented in [17] is implemented. Briefly, these arrays are extended to rectangularly contoured arrays by introducing virtual elements so that the proposed GFBM/Green's function-DFT method can be used. However, during the implementation procedure it should be assumed that currents on the virtual elements are exactly zero (i.e., $A_{n m r}=0$ for virtual elements). Therefore, in the evaluation of strong region contributions, both the voltages on these elements and all mutual couplings related to these elements are set to zero such that

$$
V_{p q s}=0 \text { if } p q \in \text { virtual elements }
$$

and

$$
Z_{n m r, p q s}=0 \text { if } n m \text { and/or } p q \in \text { virtual elements. }
$$

Implementation of (22) and (23) will assure that $A_{n m r}=0$ for virtual elements. On the other hand, in the evaluation of weak region contributions, $Z_{n m r, p q s}$ between two virtual elements and $Z_{n m r, p q s}$ between a real and a virtual element are treated the same as $Z_{n m r, p q s}$ between two real elements as opposed to (23) for strong group contributions. Notice that because the $A_{n m r}$ values of virtual elements are zero in the computation of $B_{k l r}$ values, if one employs all DFT terms, utilization of nonzero $Z_{n m r, p q s}$ when a virtual element is involved does not yield an error. However, due to the use of a few significant DFT terms, a small error might be expected. Nevertheless, such an error coming from the computation of weak region contributions does not affect the overall accuracy. 
Finally, the computational complexity and storage requirements of this method is summarized as follows: The storage requirement for the unknown current vectors, $\mathbf{I}^{f}$ and $\mathbf{I}^{b}$, during the iterative process is $2 N_{\text {tot }}$, significant DFT terms require a storage of $N_{b} Q$, where $N_{b} Q \ll N_{\text {tot }}$, and the storage requirement during the calculation of $C_{k l r, p q s}$ is $N_{\text {tot }} Q$. Meanwhile, the computational cost for the strong region is $N_{s} N_{\text {tot }}$ and $N_{s} \ll N_{\text {tot }}$, weak region calculations require an operational count of $N_{\text {tot }} Q$ and finally the computational cost for the calculations of $C_{k l r, p q s}$ and $D_{k l r, p q s}$ is $2 N_{\text {tot }} Q$. As a result, considering the fact that $Q$ and $N_{s}$ are almost constant regardless of the array size, the overall storage and computational complexity requirements of this method is $O\left(N_{\text {tot }}\right)$.

\section{NUMERICAL RESULTS AND DISCUSSION}

Numerical results in the form of array current distribution pertaining to various electrically large, rectangular and irregularly contoured two-dimensional finite arrays of probe-fed microstrip patches on grounded dielectric slabs are given to assess the efficiency and accuracy of the GFBM/Green's function-DFT method.

The first numerical example is a larger version of an array example given in [9] with the parameters $d=0.04 \lambda_{0}, \epsilon_{r}=2.55$, $L=W=0.3 \lambda_{0}, d_{x}=d_{y}=0.5 \lambda_{0},\left(x_{\text {probe }}, y_{\text {probe }}\right)=$ $(-L / 2,0)$. As the first step, results given in [9, Figs. 7 and 10] are generated with the conventional hybrid MoM/Green's function solution and excellent agreement is achieved. Then, expanding each patch using three $\hat{x}$-directed basis functions, the induced array current, $A_{n m r}$, of a $41 \times 41$ (a total of 5043 unknowns) probe-fed microstrip patch array with the aforementioned parameters is obtained with the GFBM/Green's function-DFT method and is compared with the result of the conventional MoM/Green's function method. Fig. 5(a) shows this comparison (i.e., $\left|A_{n m r}\right|$ ) for the 20th column (same as -20th column due to symmetry) when the array is excited uniformly and phased to radiate a beam maximum in the broadside direction [i.e., $\left(\theta_{i}, \phi_{i}\right)=\left(0^{\circ}, 0^{\circ}\right)$ ]. The current distribution on the first five patches are zoomed just to show the agreement with the conventional MoM/Green's function solution. Similarly, for the same array Fig. 5(b) shows the same comparison for the middle column (i.e., $m=0$ ) when the array is excited uniformly and phased to radiate a beam maximum in the direction $\left(\theta_{i}, \phi_{i}\right)=\left(20^{\circ}, 0^{\circ}\right)$. As seen from the figures, the agreement between the GFBM/Green's function-DFT and the conventional hybrid MoM/Green's function methods is very good. Desired accuracy for the GFBM/Green's function-DFT method is achieved with just three iterations by selecting $N_{s}=9(3 \times 3$ for the strong region) and using a single DFT term for each subarray (i.e., $Q=1$ and a total of three DFT terms). On the other hand, the CPU time for the GFBM/Green's function-DFT method is approximately 0.05 s/iteration, whereas the conventional MoM/Green's function method requires $1500 \mathrm{~s}$ to solve the same array.

The second numerical example is a $19 \times 19$ array version of a probe-fed microstrip patch antenna taken from [29] (case 6(b) in [29]). The substrate and the antenna dimensions for this particular antenna are given in [29] as $\epsilon_{r}=2.22, d=0.079 \mathrm{~cm}$, $L=1.25 \mathrm{~cm}, W=2 \mathrm{~cm}$ and $\left(x_{\text {probe }}, y_{\text {probe }}\right)=(0.425,0) \mathrm{cm}$.

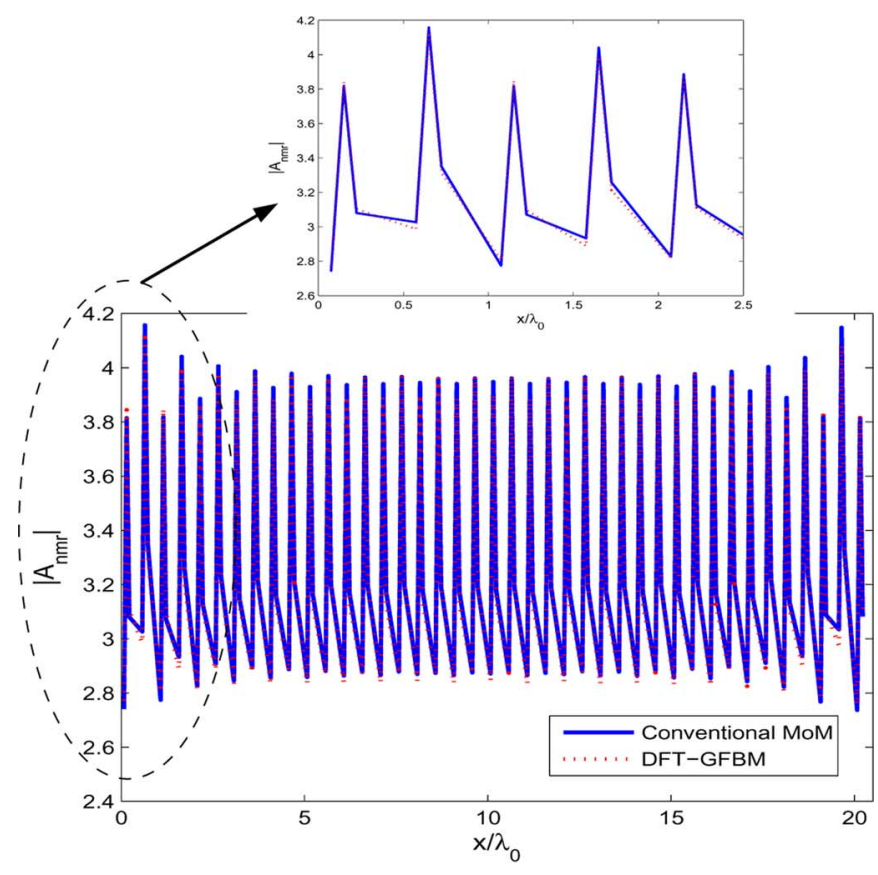

(a)

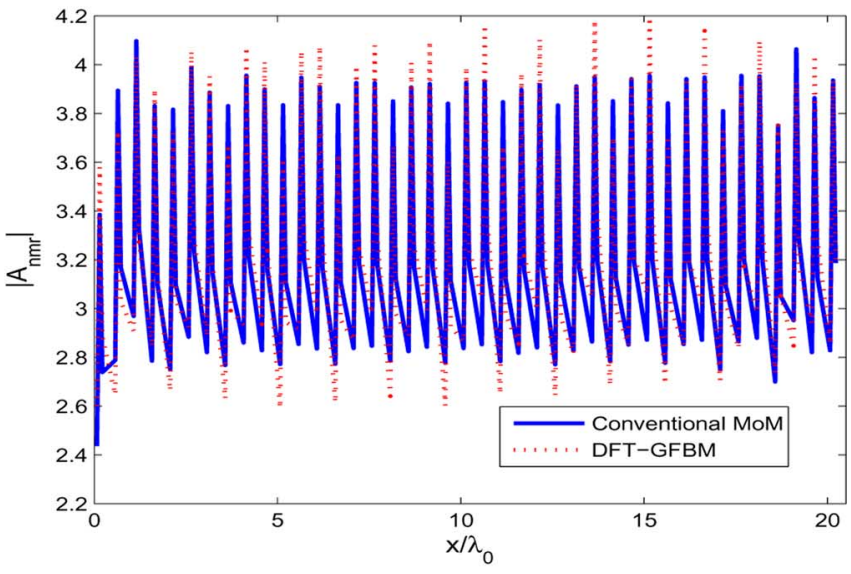

(b)

Fig. 5. Comparison of the magnitude of the induced current $\left|A_{n m r}\right|$ obtained via GFBM/Green's Function-DFT and the conventional MoM/Green's function methods for a $41 \times 41$ probe-fed microstrip patch array on a grounded dielectric slab with $d=0.04 \lambda_{0}, \epsilon_{r}=2.55$. Other parameters are: $L=W=0.3 \lambda_{0}$, $d_{x}=d_{y}=0.5 \lambda_{0}, x_{\text {probe }}=-L / 2, y_{\text {probe }}=0$. Three $\hat{x}$-directed basis functions are used for each patch. (a) 20th (or -20th) column (with with a close-up view of the current on the first 5 patches) for $\left(\theta_{i}, \phi_{i}\right)=\left(0^{\circ}, 0^{\circ}\right)$, (b) middle column for $\left(\theta_{i}, \phi_{i}\right)=\left(20^{\circ}, 0^{\circ}\right)$.

Similar to the previous example, we first simulate case 6(b) of [29] with the conventional hybrid MoM/Green's function solution using 10 basis functions (four $\hat{x}$ - and six $\hat{y}$-directed) and an excellent agreement with Fig. 8 curve b of [29] is obtained, where the frequency range is $7.3-8.5 \mathrm{GHz}$. Then, we set the frequency to $8 \mathrm{GHz}$, obtain the induced array current, $A_{n m r}$, of the $19 \times 19$ (a total of 3610 unknowns) array version of this antenna using the GFBM/Green's function-DFT method, and compare the result with that of obtained with the conventional hybrid MoM/Green's function method. In both simulations, the array is excited uniformly and phased to radiate a beam maximum in the broadside direction. Also, the interelement spacing for both simulations is selected to be $d_{x}=d_{y}=0.7 \lambda_{0}$ at $8 \mathrm{GHz}$. 


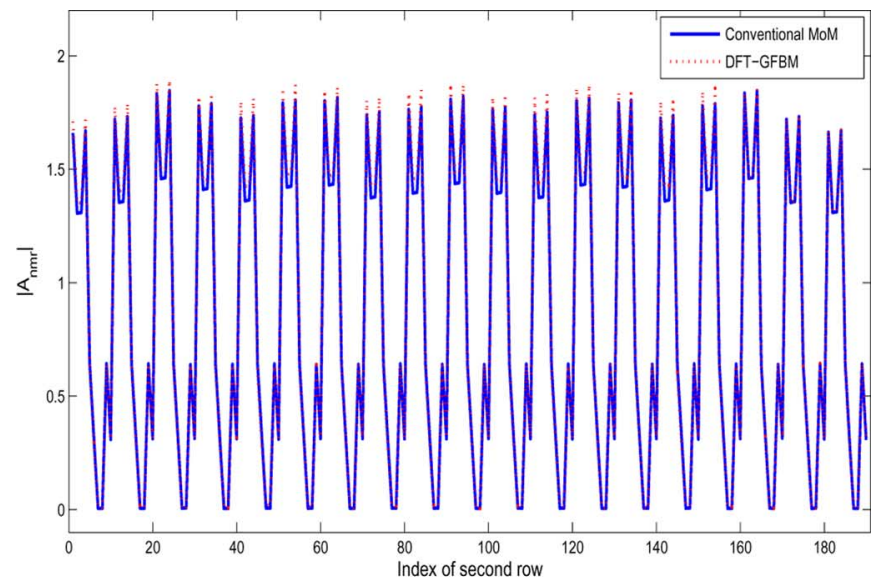

(a)

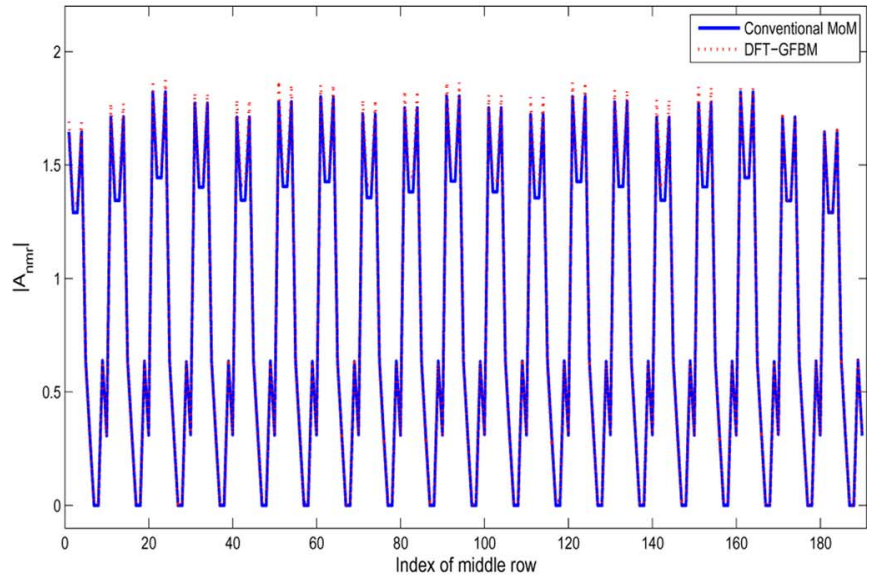

(b)

Fig. 6. Comparison of the magnitude of the induced current $\left|A_{n m r}\right|$ obtained via GFBM/Green's Function-DFT and the conventional MoM/Green's function methods for a $19 \times 19$ probe-fed microstrip patch array on a grounded dielectric slab with $d=0.021 \lambda_{0}, \epsilon_{r}=2.22$. Other parameters are: $L=0.33 \lambda_{0}$, $W=0.53 \lambda_{0}, d_{x}=d_{y}=0.7 \lambda_{0}, x_{\text {probe }}=-0.1133 \lambda_{0}, y_{\text {probe }}=0 . \mathrm{A}$ total of 10 basis functions (four $\hat{x}$ and six $\hat{y}$-directed basis functions) are used for each patch. (a) 8th (or -8 th) column, (b) middle column.

Fig. 6(a) and (b) show this comparison (i.e., $\left|A_{n m r}\right|$ ) for the 8th (or the -8 th) and middle columns, respectively, where results agree with each other very well. Similar to the previous example, desired accuracy for the GFBM/Green's function-DFT method is achieved again within three iterations by selecting $N_{s}=9(3 \times 3$ for the strong region) and $Q=1$ (a total of 10 DFT terms). Finally, the elapsed CPU time to solve this array with the conventional MoM/Green's function method is approximately $532 \mathrm{~s}$, whereas it is only $0.1 \mathrm{~s} /$ iteration for the GFBM/Green's function-DFT method.

As a third example using the parameters of Fig. 5, a 1257-element (a total of 3771 unknowns) probe-fed microstrip patch antenna array with a circular boundary is considered. The size of the corresponding rectangular array is $41 \times 41$ after the virtual elements are introduced. Fig. 7(a) and (b) show a comparison of the magnitude of the induced array current, $\left|A_{n m r}\right|$, for the 16th (or -16 th) and 1st (i.e., $m=1$, or -1 st) columns, respectively. In both simulations the array is scanned in the broadside direction. As in the case of the first numerical example, very good

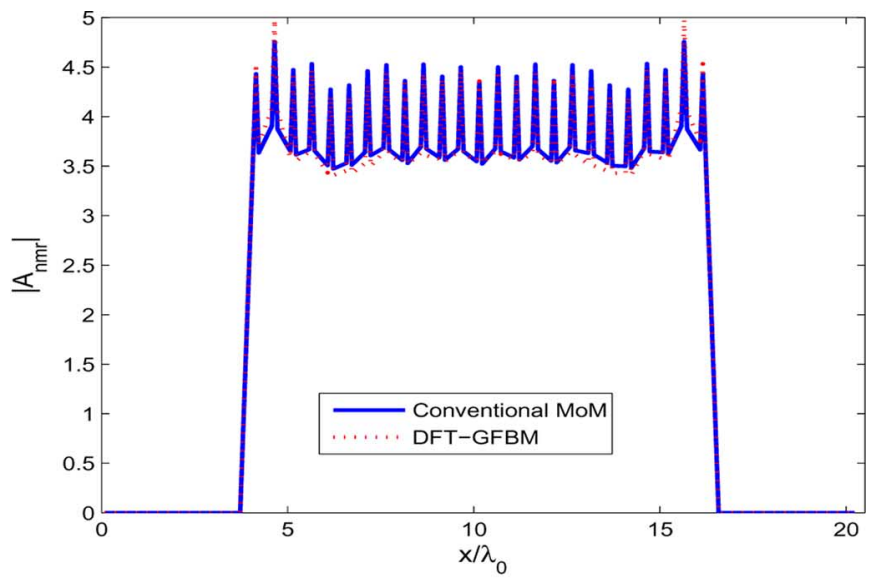

(a)

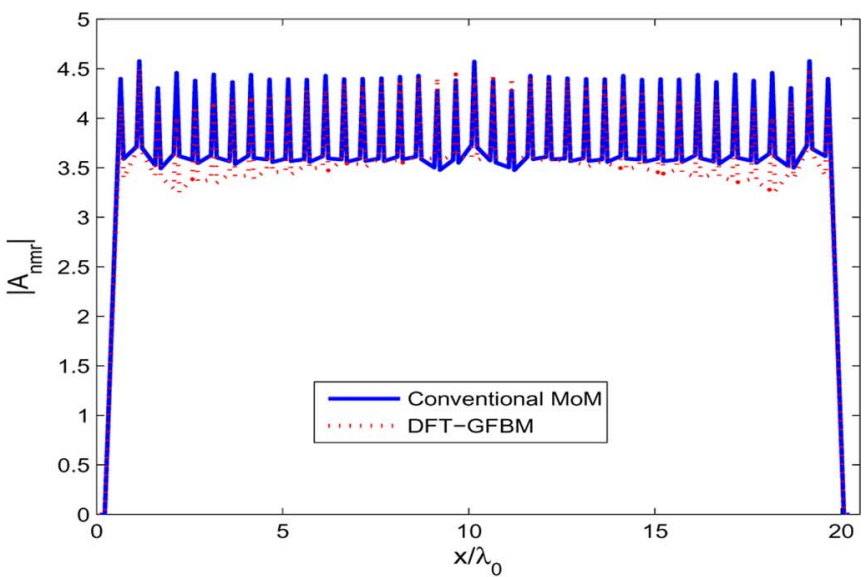

(b)

Fig. 7. Comparison of the magnitude of the induced current $\left|A_{n m r}\right|$ obtained via GFBM/Green's Function-DFT and the conventional MoM/Green's function methods for a 1257 -element $(41 \times 41)$ circular, probe-fed microstrip patch array on a grounded dielectric slab. All parameters are the same as in Fig. 5. (a) 16th (or -16 th) column, (b) 1st (or -1 st) column.

agreement between GFBM/Green's function-DFT and the conventional MoM/Green's function methods is achieved within three iterations by selecting $N_{s}=9$ and $Q=3$. Just like the previous two examples, a significant acceleration is achieved for the solution of the array with the GFBM/Green's function-DFT method. While the conventional MoM/Green's function method solves this array in $630 \mathrm{~s}$, it takes only 0.09 s/iteration for the GFBM/Green's function-DFT solution.

Our final example is the elliptical version of the example given in Fig. 7 composed of 843 probe-fed microstrip patch antennas (a total of 2529 unknowns). The size of the corresponding rectangular array is $43 \times 27$ after the virtual elements are introduced. Fig. 8(a) and (b) show a comparison of the magnitude of the induced array current, $\left|A_{n m r}\right|$, for the 10th (or -10 th) and middle columns, respectively for a broadside scan. Similar to the previous example, within three iterations with $N_{s}=9$ and $Q=3$, very good agreement between GFBM/Green's function-DFT and the conventional MoM/Green's function methods is achieved. Moreover, similar to the previous examples, a significant acceleration is achieved for the solution of the array with the GFBM/Green's function-DFT approach. It takes 185 


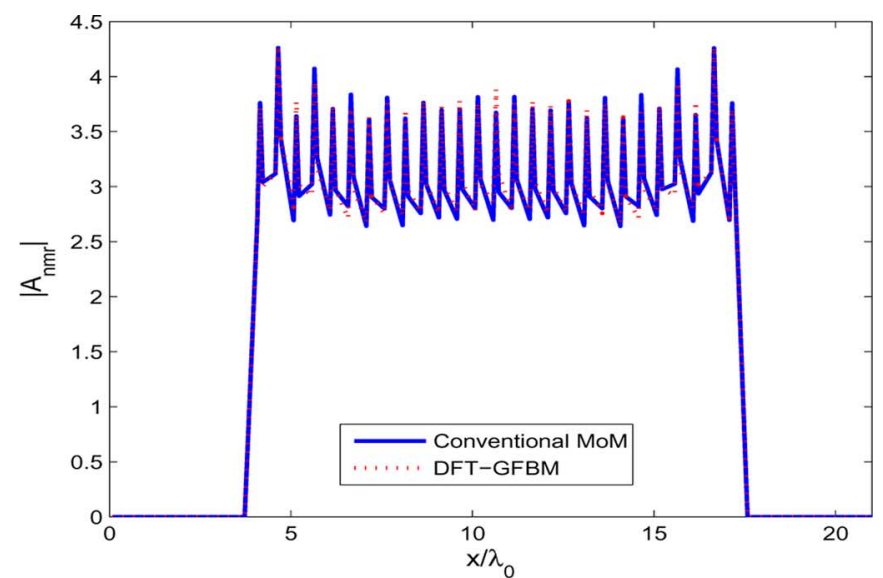

(a)

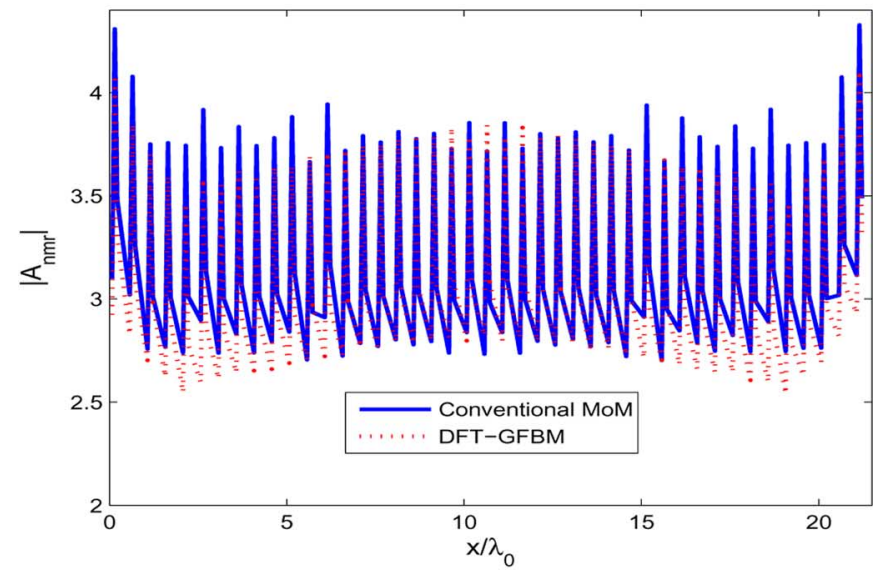

(b)

Fig. 8. Comparison of the magnitude of the induced current $\left|A_{n m r}\right|$ obtained via GFBM/Green's Function-DFT and the conventional MoM/Green's function methods for a 843 -element $(43 \times 27)$ elliptical, probe-fed microstrip patch array on a grounded dielectric slab. All parameters are the same as in Fig. 5. (a) 10th (or -10 th) column, (b) middle column.

$\mathrm{s}$ for the conventional MoM/Green's function method to solve this elliptically contoured array, whereas only 0.06 s/iteration is enough to solve the same array with the GFBM/Green's function-DFT method.

Finally it should be mentioned that all numerical results are obtained using a Pentium IV $3.2 \mathrm{GHz}$ personal computer, and in all these numerical examples the percentage error defined as

$$
\text { Error }=\frac{\left\|\mathbf{I}_{\mathrm{MoM}}-\mathbf{I}_{\mathrm{GFBM}-\mathrm{DFT}}\right\|}{\left\|\mathbf{I}_{\mathrm{MoM}}\right\|} \times 100
$$

where $\mathbf{I}_{\mathrm{MoM}}$ and $\mathbf{I}_{\mathrm{GFBM}-\mathrm{DFT}}$ pertain to $\left[A_{n m r}\right]_{N_{\mathrm{tot}} \times 1}$ column vectors obtained using the conventional MoM/Green's function and GFBM/Green's function-DFT methods, respectively, is less than 5\%. In (24), $\|\cdot\|$ stands for the Frobenius norm. Actually a slight increase in the strong region or in the number of DFT terms decreases this error further.

\section{CONCLUSION}

A novel method, named GFBM/Green's function-DFT method, is presented for the fast and accurate analysis of arbitrarily contoured finite phased arrays of probe-fed microstrip patches on planar grounded dielectric slabs. It is based on a hybrid combination of the GFBM with the grounded dielectric slab's Green's function and its acceleration with a DFT based algorithm. The method can also handle arrays with arbitrarily shaped elements since the first step of the method is to find the current coefficients of a single patch by employing a conventional Galerkin type hybrid MoM/Green's function method, where an arbitrary number of subsectional basis functions can be used. The computational complexity of this method is $O\left(N_{\text {tot }}\right)$ for each iteration, which is achieved by combining a generalized version of the DFT based acceleration algorithm developed in [14] with the fact that array elements are identical and the array is periodic.

Several numerical examples, in the form of array current distribution, are provided for various arrays with rectangular, circular and elliptical boundaries and results are compared with a reference solution based on the conventional hybrid MoM/ Green's function method to assess the efficiency and accuracy of this method. Very good agreement is observed between the hybrid GFBM/Green's function-DFT and the reference solutions for each array. Furthermore, as expected, a great reduction in the solve time of these arrays is achieved. Work is in progress to extend this method to multilayer geometries.

\section{REFERENCES}

[1] D. T. McGrath and W. P. Green, "Phased array antenna analysis with hybrid finite element method," IEEE Trans. Antennas Propag., vol. 42, pp. 1625-1630, Dec. 1994.

[2] R. W. Kindt, K. Sertel, E. Topsakal, and J. L. Volakis, "Array decomposition method for the accurate analysis finite arrays," IEEE Trans. Antennas Propag., vol. 51, pp. 1364-1372, Jun. 2003.

[3] J. Rubio, M. A. Gonzalez, and J. Zapata, "Generalized-scattering-matrix analysis of a class of finite arrays of coupled antennas by using 3-D FEM and spherical mode expansion," IEEE Trans. Antennas Propag., vol. 53, pp. 1133-1144, Mar. 2005.

[4] G. M. Turner and C. Christodoulou, "FDTD analysis of phased array antennas," IEEE Trans. Antennas Propag., vol. 47, pp. 661-667, Apr. 1999.

[5] A. Ishimaru, R. J. Coe, G. E. Miller, and W. P. Green, "Finite periodic structure approach to large scanning array problems," IEEE Trans. Antennas Propag., vol. 33, pp. 1213-1220, Nov. 1985.

[6] A. K. Skrivervik and J. R. Mosig, "Finite phased array of microstrip patch antennas: The infinite array approach," IEEE Trans. Antennas Propag., vol. 40, pp. 579-582, May 1992.

[7] A. K. Skrivervik and J. R. Mosig, "Analysis of finite phased arrays of microstrip patches," IEEE Trans. Antennas Propag., vol. 41, pp. 1105-1114, Aug. 1993.

[8] D. M. Pozar, "Analysis of finite phased arrays of printed dipoles," IEEE Trans. Antennas Propag., vol. 33, pp. 1045-1053, Oct. 1985.

[9] D. M. Pozar, "Finite phased arrays of rectangular microstrip patches," IEEE Trans. Antennas Propag., vol. 34, pp. 658-665, May 1986.

[10] H.-T. Chou, H.-K. Ho, O. A. Civi, and V. B. Ertürk, "Applications of hybrid discrete Fourier transform-moment method to the fast analysis of large rectangular dipole arrays printed on a thin grounded dielectric substrate," Microw. Opt. Technol. Lett., vol. 34, no. 3, pp. 203-207, Aug. 2002.

[11] H.-T. Chou, H.-K. Ho, P. H. Pathak, P. Nepa, and O. A. Civi, "Efficient hybrid discrete Fourier transform-moment method for fast analysis of large rectangular arrays," Proc. Inst. Elect. Eng. Microwave Antennas Propag., vol. 149, no. 1, pp. 1-6, Feb. 2002.

[12] P. Janpugdee, "An efficient discrete Fourier transform based ray analysis of large finite planar phased arrays," M.S. thesis, Dept. of Electrical Engineering, The Ohio-State University, Columbus, 2002.

[13] P. Janpugdee and P. H. Pathak, "A DFT-based UTD ray analysis of large finite phased arrays on a grounded substrate," IEEE Trans. Antennas Propag., vol. 54, pp. 1152-1161, Apr. 2006. 
[14] H.-T. Chou and H.-K. Ho, "Implementation of a forward-backward procedure for the fast analysis of electromagnetic radiation/scattering from two-dimensional large phased arrays," IEEE Trans. Antennas Propag., vol. 52, pp. 388-396, Feb. 2004.

[15] Ö. A. Civi, "Extension of forward backward method with DFT based acceleration algorithm for the efficient analysis of radiation/scattering from large finite printed dipole arrays," Microw. Opt. Technol. Lett., vol. 37, no. 1, pp. 20-26, Apr. 2003.

[16] V. B. Ertürk and H. T. Chou, "Efficient analysis of large phased arrays using iterative MoM with DFT-based acceleration algorithm," Microwave Opt. Technol. Lett., vol. 39, no. 2, pp. 89-94, Oct. 2003.

[17] Ö. A. Civi, V. B. Ertürk, and H. T. Chou, "Extension of forward backward method with DFT based acceleration algorithm for the efficient analysis of large periodic arrays with arbitrary boundaries," Microw. Opt. Technol. Lett., vol. 47, no. 3, pp. 293-298, Nov. 2005.

[18] Y. Zhuang, K.-L. Wu, C. Wu, and J. Litva, "A combined full-wave CG-FFT method for rigorous analysis of large microstrip antenna arrays," IEEE Trans. Antennas Propag., vol. 44, pp. 102-109, Jan. 1996.

[19] C.-F. Wang, F. Ling, and J.-M. Jin, "A fast full-wave analysis of scattering and radiation from large finite arrays of microstrip antennas," IEEE Trans. Antennas Propag., vol. 46, pp. 1467-1474, Oct. 1998.

[20] P. D. Vita, A. Freni, F. Vipiana, P. Pirinoli, and G. Vecchi, "Fast analysis of large finite arrays with a combined multiresolution-SM/AIM approach," IEEE Trans. Antennas Propag., vol. 54, pp. 3827-3832, Dec. 2006.

[21] Ö. A. Civi, V. B. Ertürk, P. H. Pathak, P. Janpugdee, and H. T. Chou, "A hybrid UTD-MoM approach for the efficient analysis of radiation/ scattering from large, printed finite phased arrays," in Proc. IEEE APS Int. Symp. and URSI Radio Science Meeting, Boston, MA, Jul. 2001, vol. 2, pp. 806-809.

[22] Ö. A. Civi, P. H. Pathak, H.-T. Chou, and P. Nepa, "A hybrid uniform geometrical theory of diffraction-moment method for the efficient analysis of electromagnetic radiation/scattering from large finite planar arrays," Radio Sci., vol. 35, no. 2, pp. 607-620, Mar.-Apr. 2000.

[23] A. Neto, S. Maci, G. Vecchi, and M. Sabbadini, "A truncated Floquet wave diffraction method for the full-wave analysis of large phased arrays-Part 2: Generalization to 3D cases," IEEE Trans. Antennas Propag., vol. 48, pp. 601-611, Apr. 2000.

[24] M. Pino, L. Landesa, J. L. Rodriguez, F. Obelleiro, and R. J. Burkholder, "The generalized forward-backward method for analyzing the scattering from targets on ocean-like rough surfaces," IEEE Trans. Antennas Propag., vol. 47, pp. 961-968, Jun. 1999.

[25] S. Barkeshli, "An efficient approach for evaluating the planar microstrip Green's function and its applications to the analysis of microstrip antennas and arrays," Ph.D. dissertation, Dept. Electrical Engineering, The Ohio-State University, Columbus, 1988.

[26] M. Marin, S. Barkeshli, and P. H. Pathak, "Efficient analysis of planar microstrip geometries using a closed-form asymptotic representation of the grounded dielectric slab Green's function," IEEE Trans. Antennas Propag., vol. 37, pp. 669-679, Apr. 1989.

[27] S. Barkeshli, P. H. Pathak, and M. Marin, "An asymptotic closed-form microstrip surface Green's function for the efficient moment method analysis of mutual coupling in microstrip antennas," IEEE Trans. Antennas Propag., vol. 38, pp. 1374-1383, Sep. 1990.

[28] O. Bakır, "Investigation of Finite phased arrays of printed antennas on planar and cylindrical grounded dielectric slabs," M.Sc. thesis, Dept. of Electrical and Electronics Engineering, Bilkent University, Turkey, 2006.

[29] D. H. Schaubert, D. M. Pozar, and A. Adrian, "Effect of microstrip antenna substrate thickness and permittivity: Comparison of theories with experiment," IEEE Trans. Antennas Propag., vol. 37, pp. 677-682, Jun. 1989.

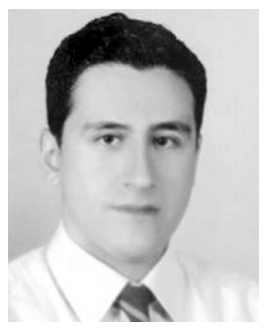

Onur Bakır (S'05) was born in Kayseri, Turkey, in 1980. He received the B.S. and M.S. degrees in electrical engineering from Bilkent University, Bilkent, Turkey in 2003 and 2006, respectively. He is currently working towards the Ph.D. degree at the University of Michigan, Ann Arbor.

His research interests include computational electromagnetics, design and analysis of microstrip antennas on planar and curved surfaces.

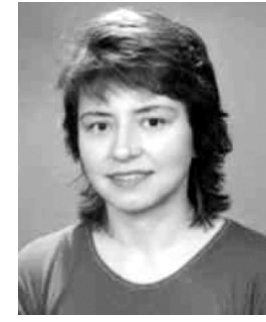

Özlem Aydın Çivi (S'90-M'97-SM'05) received B.Sc., M.Sc., and Ph.D. degrees in electrical and electronics engineering from the Middle East Technical University (METU), Ankara, Turkey, in 1990, 1992, and 1996, respectively.

From 1990 to 1996, she was a Research Assistant at METU. From 1997 to 1998, she was a Visiting Scientist at the ElectroScience Laboratory, Ohio State University. Since 1998, she has been with the Department of Electrical and Electronics Engineering, METU, where she is currently an Associate Professor. Her research interests include analytical, numerical and hybrid techniques in EMT problems, especially fast asymptotic/hybrid techniques for the analysis of large finite periodic structures, multifunction antenna design, phased arrays and RF-MEMS applications. Since 1997, she is a National Expert of the European actions COST260, COST284, COST-IC0603 on antennas. She is one of the Work Package Leaders of AMICOM, a European Network of Excellence on RF-MEMS. Since 2004, she is a Technical Reviewer of the European Community for scientific projects in the fields of antennas and communication. She has published over 80 journal and international conference papers.

Dr. Çivi was a recipient of the 1993 Erol Gelenbe Best Masters Thesis award, the 1994 Prof. Mustafa Parlar Foundation Research and Encouragement award with the METU Radar Group, and the 1996 URSI Young Scientist award. She was the Chair of the IEEE AP/MTT/ED/EMC Chapter between 2004 to 2006 and the Chair of the IEEE Turkey Section between 2006 to 2007. She is a member of the Administrative Committee of the Turkish National Committee of URSI.

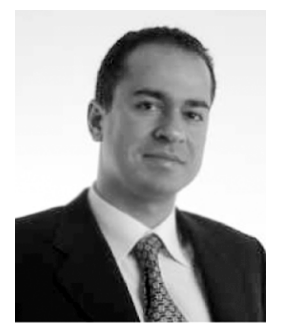

Vakur B. Ertïrk (M'00) received the B. S. degree in electrical engineering from the Middle East Technical University, Ankara, Turkey, in 1993, and the M. S. and Ph.D. degrees from The Ohio State University (OSU), Columbus, in 1996 and 2000, respectively.

$\mathrm{He}$ is currently an Assistant Professor with the Electrical and Electronics Engineering Department, Bilkent University, Ankara. His research interests include the analysis and design of planar and conformal arrays, active integrated antennas, scattering from and propagation over large terrain profiles as

well as metamaterials.

Dr. Ertürk served as the Secretary/Treasurer of the IEEE Turkey Section as well as the Turkey Chapter of the IEEE Antennas and Propagation, Microwave Theory and Techniques, Electron Devices and Electromagnetic Compatibility Societies. He was the recipient of the 2005 URSI Young Scientist Award and the 2007 Turkish Academy of Sciences Distinguished Young Scientist Award.

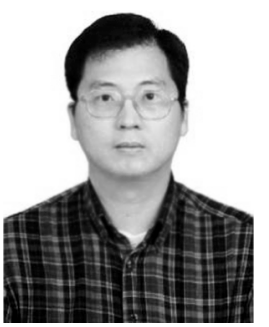

Hsi-Tseng Chou (SM'00) was born in Taiwan, R.O.C., in 1966. He received the B.S. degree in electrical engineering from National Taiwan University, Taiwan, R.O.C., in 1988, and the M.S. and Ph.D. degrees in electrical engineering from the Ohio State University (OSU), Columbus, in 1993 and 1996, respectively.

After completing his military obligation, he spent one year at China Raydon Corp., as an R\&D Engineer, during which time he was sent to Mitsubishi Electronic in Japan for three months' technical training. He joined the ElectroScience Laboratory (ESL), OSU as a Graduate Research Associate during 1991 to 1996 and as a Postdoctoral Researcher during 1996 to 1998. He joined Yuan-Ze University (YZU), Taiwan, R.O.C., in August 1998, and is currently a Professor in the Department of Communications Engineering. While at YZU, he was simultaneously a Visiting Scholar at Far Eastone Telecomm for five years from 1999 to 2004. His research interests include wireless communication network, antenna design, antenna measurement, electromagnetic scattering, asymptotic high frequency techniques such as uniform geometrical theory of diffraction (UTD), novel Gaussian beam techniques, and UTD type solution for periodic structures. He has published more than 170 journal and conference papers.

Dr. Chou is an elected member of URSI International Radio Science US commission B. He received many awards to recognize his distinguished contributions in technological development. In 1999, he received a Young Scientist 
Award from URSI and a Best Paper Award from the OSU-ESL. In 2002, he received a Young Scientist Research Award from Academia Sinica of Taiwan and a Distinguished Research Award from YZU. In 2003, he received an award from the Education Ministry for his "Distinguished Contribution in Promoting Interacademic and Industrial Cooperation," and a Young Electrical Engineer Award from the Chinese Institute of Electrical Engineering. The commercial products resulting from his cooperation with industries have been awarded twice in 1998 and 2003, respectively, by the Economic Ministry as "Taiwan Symbols of Excellence," and elected as one of the 11 distinguished commercial products in Hsin-Chu Science Part of Taiwan in 2003. As a result, the cooperating industry had been awarded the "Fast Growth of the Export and Import in the International Trade" in 2002 by the Economic Ministry due to the successful commercialization, and YZU received a Distinguished Inter-Academic and Industrial Cooperation Award from the Chinese Institute of Engineers in 2004. Also in 2004 he received a Distinguished Engineering Professor Award from the Chinese Institute of Engineers, a Distinguished Service Award from YZU, and he was elected as one of the nation's Ten Outstanding Young Persons by the Junior Chamber International. In 2005, he received a National Young Person Medal from the China Youth Corps of Taiwan and a Distinguished Professor Award from Y. Z. Hsu Science and Technology Memory Foundation. In 2006, he was elected as one of the Top 10 Rising Stars in Taiwan by the Central News Agency of Taiwan, and was awarded a position of Chair Professor by YZU and the Y. Z. Hsu Science and Technology Memory Foundation, which makes him the youngest chair professor in YZU's history. He was placed in the Outstanding Category in the YZU Annual Research Evaluation since 1998, and received several research awards from the National Science Council. 